\title{
Local Heat Dissipation of Ag Nanowire Networks Examined with Scanning Thermal Microscopy
}

Seunghoe Koo ${ }^{1}$, Jaehee Park ${ }^{1}$, Sangmo Koo ${ }^{* 1}$ and Kyeongtae Kim ${ }^{*}, 1$

Supporting Information

\section{Supporting Information. 1 Quantitative measurement method in air}

Since the silver nanowire(AgNW) device is typically operated in air, in order to measure the thermal characteristic in the environment in which it operates, despite of the difficulty in measurement due to air convection, we applied the quantitative measurement method reported by Kim et $\mathrm{al}^{\mathrm{S} 1}$. Figure S1a shows a schematic diagram of the experimental setup for the active-mode method. As Kim et al ${ }^{\mathrm{S} 1}$. reported, using the passive mode (Figure S1b), which measures the heat transferred from the sample to the probe, and the active mode (Figure S1c), which measures the heat escaping from the heated probe to the sample, the relationship between the temperature of the sample and that of the probe could be defined experimentally. To quantitatively measure the breakdown process of the AgNW, the following experimental process was carried out. First we needed to find a spot with the highest temperature in the nanowire network. This spot has the highest probability that a breakdown will occur first. Thus, a small voltage that could not destroy the nanowire was applied to the silver nanowire network, which was then scanned using SThM to 
find the point with the highest temperature (passive mode). After that, at the point with the highest temperature, the temperature of the probe during contact and non-contact was measured at various applied voltages. When a voltage with a very fast frequency is applied to a Wheatstone bridge circuit, the probe can be heated to the desired temperature (active mode). Finally, the values required for the temperature relationship were derived. The relationship between the temperature of the sample and that of the probe is described using the equation below:

$$
T_{s}=T_{c}+\varphi\left(T_{n c}-T_{c}\right) .
$$

In the equation, $T_{s}$ is the temperature of the sample, $T_{n c}$ is the probe temperature just before the probe contacts the sample (non-contact), $T_{c}$ is the probe temperature at the time of contact, and $\varphi$ is a dimensionless proportionality constant including the thermal contact resistance property between the probe and the sample. The heat transfer phenomenon generated at the same point is maintained, and because the temperature of the probe is proportional to the temperature of the sample, it is possible to quantitatively measure the temperature of the sample at that point by obtaining $\varphi$. In this study, the experimental values of $\varphi=45.496 \mathrm{~K} / \mathrm{K}$ (Figure S2) and $T_{c}-T_{n c}=$ $0.0221 \cdot T_{c}$ were obtained. Using these values, the final proportional relationship between the temperature of the sample and that of the probe is as follows:

$$
T_{s}=2.005 \cdot T_{c} .
$$

Using the above equation, the breakdown temperature of the AgNWs was measured by gradually increasing the voltage applied to the AgNW network. This limited quantitative measurement method has also been utilized for thermal profile of graphene devices ${ }^{\mathrm{S} 2}$. 


\section{Supporting Information. 2 Fabrication of thin-film gold specimens to induce the local Joule heating phenomenon}

Owing to the limitation of the spatial measurement range of SThM $(50 \mu \mathrm{m} \times 50 \mu \mathrm{m})$, it was necessary to induce the phenomenon occurring in the nanowire at the desired point. The two Au electrodes were fabricated $\sim 10 \mu \mathrm{m}$ apart to create an area where only nanowires exist. Since the resistance of the gold electrodes is much lower than that of the AgNW channel, the current flowed almost exclusively from the gold film to the electrode gap where the gold pattern existed. In the area without gold patterns, current flowed through the coated nanowires. Therefore, the phenomenon was induced in the desired area as we controlled the gap size between the electrodes (Figure S3). The manufacturing process for the sample began with a thermal growth of $\sim 1 \mu \mathrm{m}$ silicon oxide on a silicon wafer. Then, Au lines ( $\mathrm{Cr} / \mathrm{Au}$ : $5 / 50 \mathrm{~nm})$ were created by photolithography, metal evaporation, and liftoff. Finally, AgNWs were coated on the sample through spin coating and then the sample was annealed at $140{ }^{\circ} \mathrm{C}$ in air for $40 \mathrm{~min}$. 


\section{Supporting Information. 3 another case of the nanowire breakdown process}

We investigated the results for the another case of the phenomenon shown in the manuscript. In the Figure S4, the main electrical path of the nanowire network changed, as reported by Das et al ${ }^{\mathrm{S} 3}$. Figure S4a,b show the results of measuring the applied power, resistance, current, and temperature rise against the voltage applied to the nanowire. As in the manuscript, the breakdown phenomena were divided into three parts; (i) stable heating, (ii) another channel electrical connection, and (iii) after temperature drop. The schematics for each phenomenon was expressed in Figure S4c-e. Section (i) involved stable heating and section (ii) is a process in which other channels are electrically connected and the resistance decreases slightly (matched with the case in manuscript). In addition, when $1.4 \mathrm{~V}$ was applied, a temperature decrease was found at a relatively low temperature with a slight increase in resistance. After the temperature decreased, as the applied voltage gradually increased, the temperature increased. Generating the temperature rise again meant that the nanowire channel was not completely broken. This result indicates that the resistance increased owing to the damage of the channel being monitored, leading to a decrease in the current flowing and in the temperature of the corresponding channel (Figure S4e). In addition, in section (iii), although the temperature rise decreased, the applied power of the network did not show a significant difference. This represents that the electrons were mainly transmitted by a channel other than the one being monitored. Thus, the main channel that mainly transferred the electrons in the nanowire network changed. The results in the second case show that the main channel that carried electrons in the nanowire network could change between actions. 
Supporting Information. 4 Finite element modeling (FEM) performed to simulate the effect of current concentration and Joule heating.

We investigated the current density profile generated when current flows through the nanowire junction and the temperature distribution due to Joule heat using FEM. Jeong et $\mathrm{al}^{\mathrm{S}}{ }^{\text {. reported that }}$ electromigration occurs where the current density is concentrated through FEM. Figure S5a shows the geometry of the nanowires used in the analysis. Two nanowires on the silicon oxide film were bonded to each other, and the current flowed through the junction. Figures S5b,c show the results of simulations of the current density distribution and temperature distribution in the nanowire channel, respectively. Figure S5b shows that the current density was the highest at the nanowire junction. This indicates that the movement of electrons was spatially limited, and that electromigration occurred in the junction. Figure S5c shows that the highest temperature was observed at the nanowire junction, with only a $7 \%$ difference from the surface temperature. As the breakdown temperature measured using SThM in the main text refers to the temperature at the surface of the nanowire, the temperature at the junction at which breakdown occurs is calculated as $465 \mathrm{~K}$. The values of the parameters used in the simulations are listed in Table 1. 
(a)

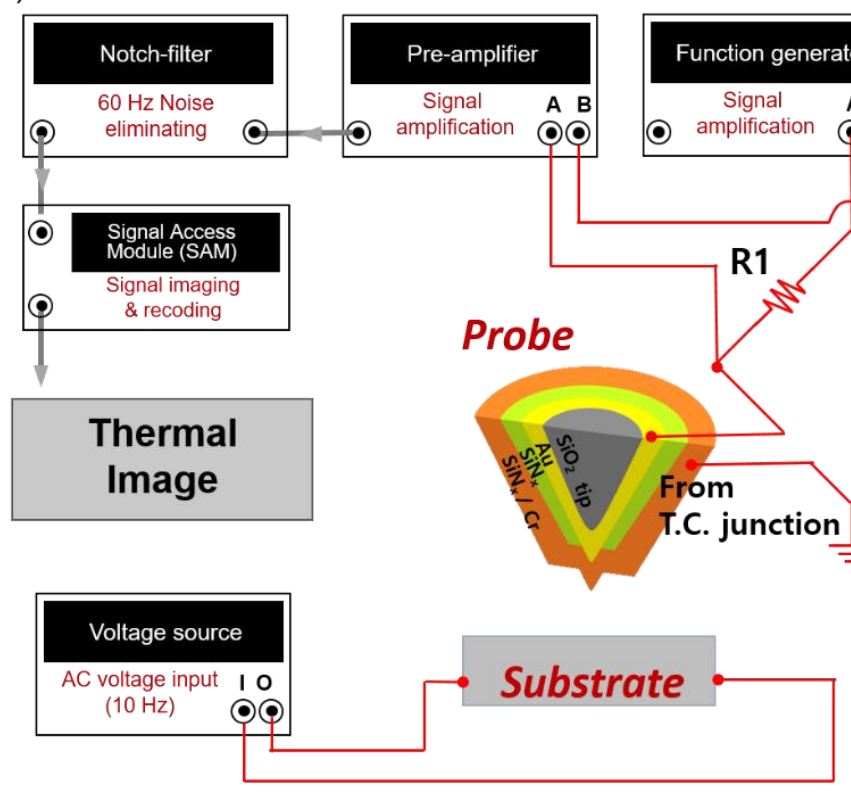

(b) < Passive mode >

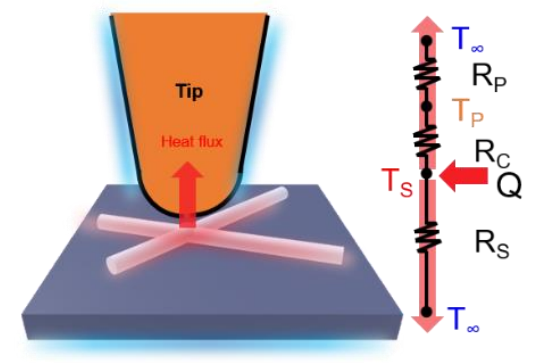

(c) < Active mode >

R3

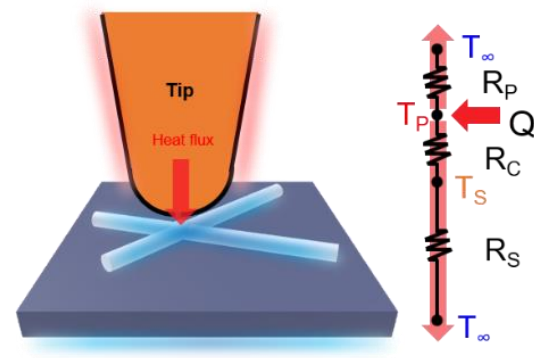

Figure S1. A method for quantitative temperature measurement in air. (a) Measurement circuit diagram using a scanning thermal probe composed of an amplifier and a noise filter for precise measurement. A schematic diagram of passive and active modes and a thermal resistance network model, respectively (b), (c). Measuring the amount of heat exchanged between the probe and the sample to obtain a quantitative thermal property value at that point. 


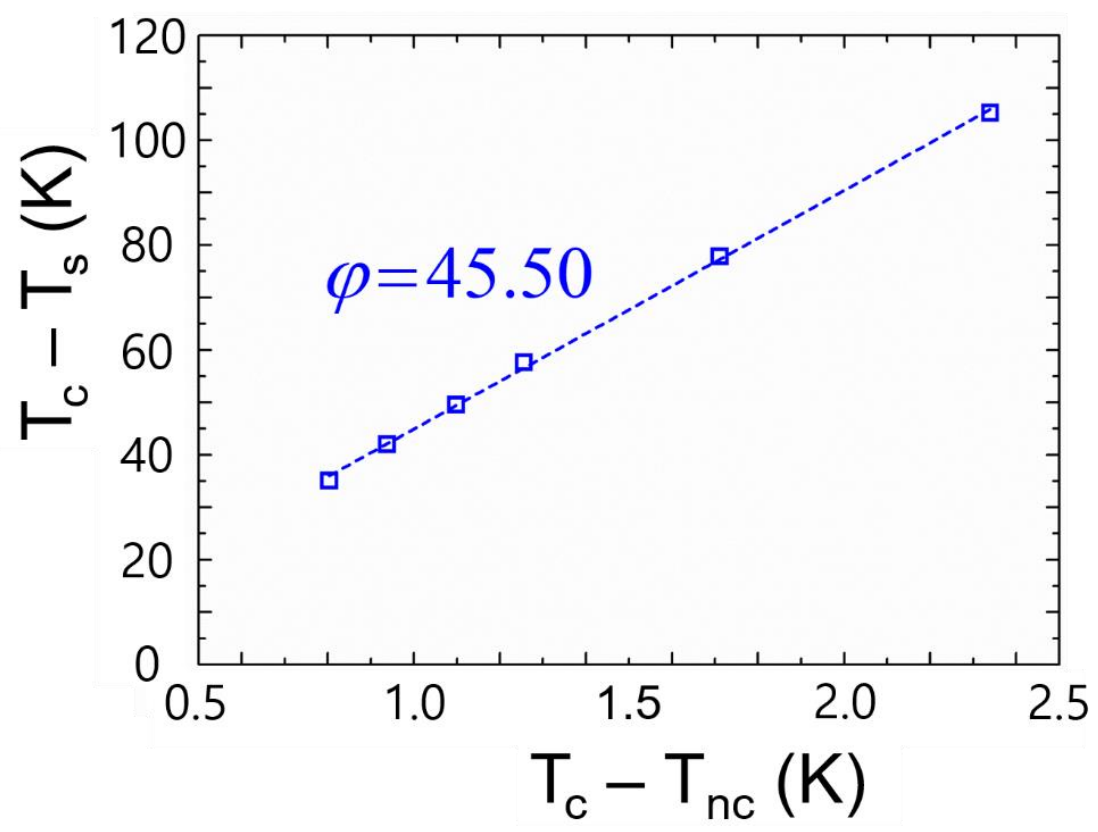

Figure S2. The dimensionless proportionality constant $(\varphi)$ that describes the thermal contact resistance property between the probe and the sample.

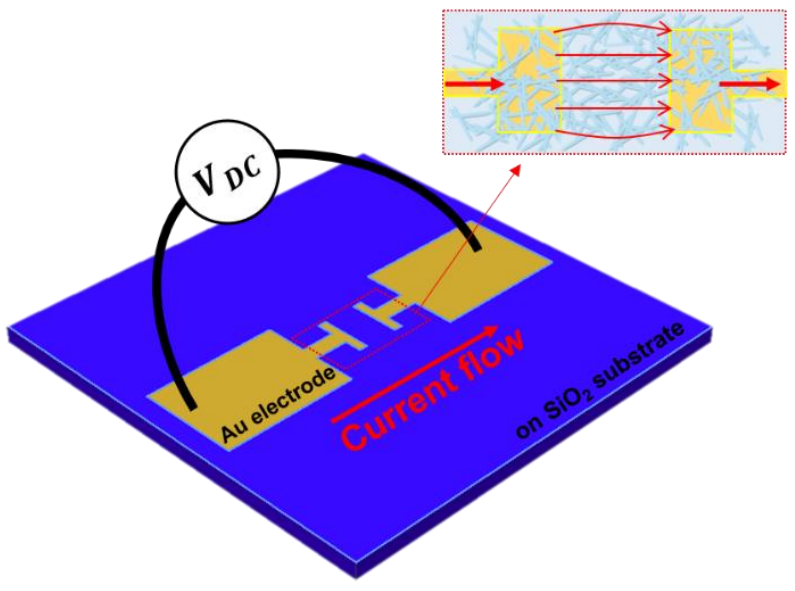

Figure S3. Restriction of nanowire electrical path area through sample fabrication. 
(a)

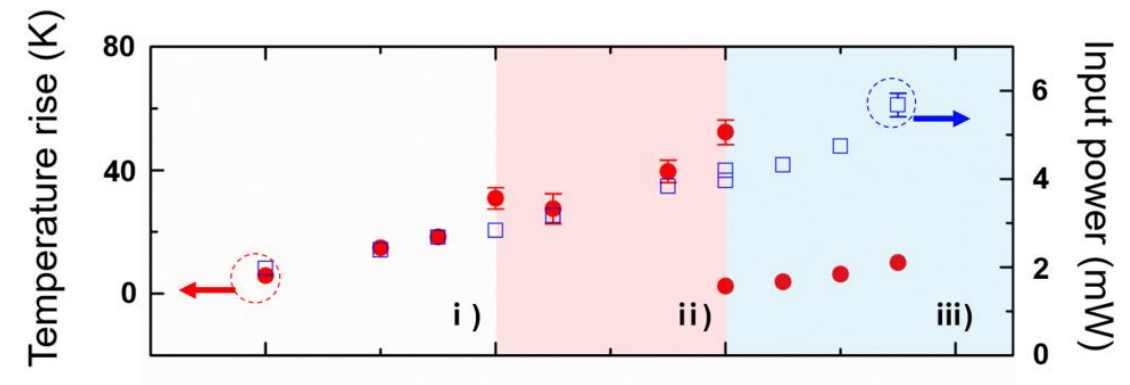

(b)
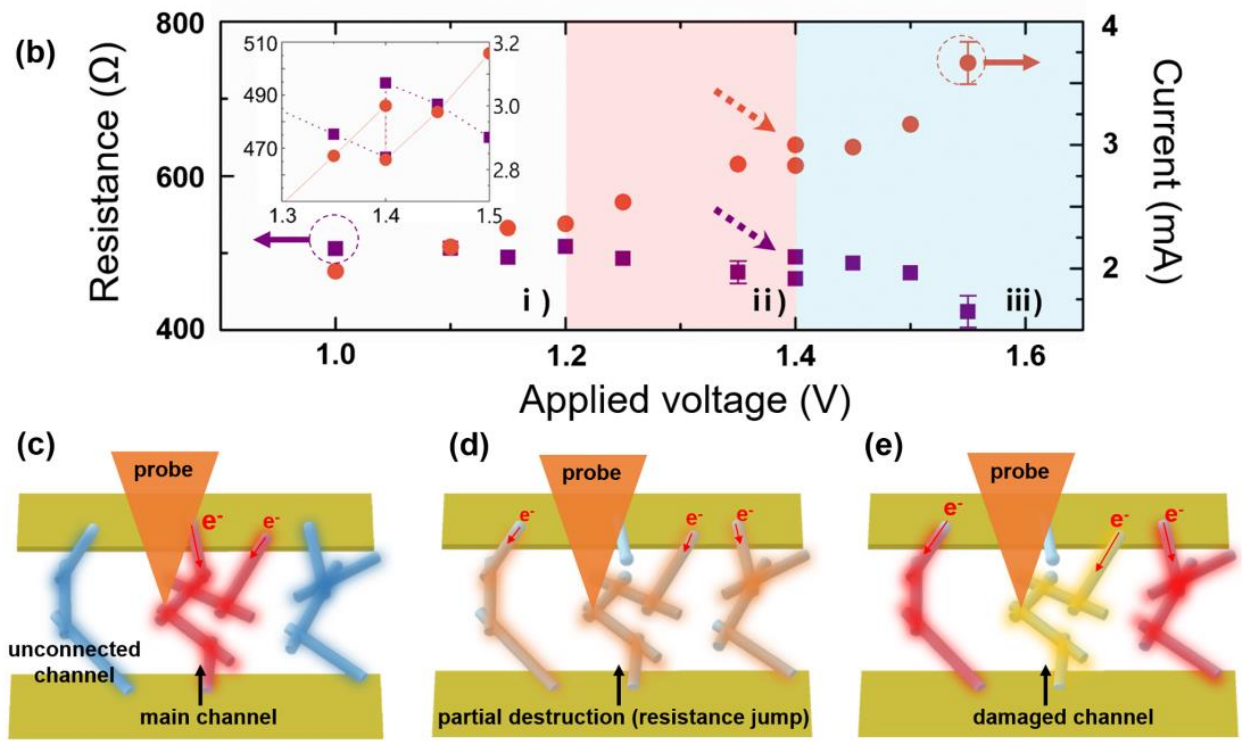

Figure S4. Measurements of the destruction phenomena of the nanowire for the second case. (a) Local temperature rise and total power applied to the network according to the applied voltage. (b) Total resistance and total current of the network according to the applied voltage. Graphs are also divided into i) stable heating, ii) electrical connection of another channel, and iii) after damage. (c-e) Schematic diagrams showing the phenomenon that occurs in each section. When the applied voltage was $1.4 \mathrm{~V}$, the temperature rise reduced because the nanowire was damaged. After that, unlike the result in the manuscript case, the temperature rose again as the voltage increased. The inset shows the electrical resistance and current when the voltage was $1.3-1.5 \mathrm{~V}$ (The area indicated by the orange and purple dashed arrow); the electrical resistance had a slight increase $(\sim 5 \%)$ at $1.4 \mathrm{~V}$. 
(a)

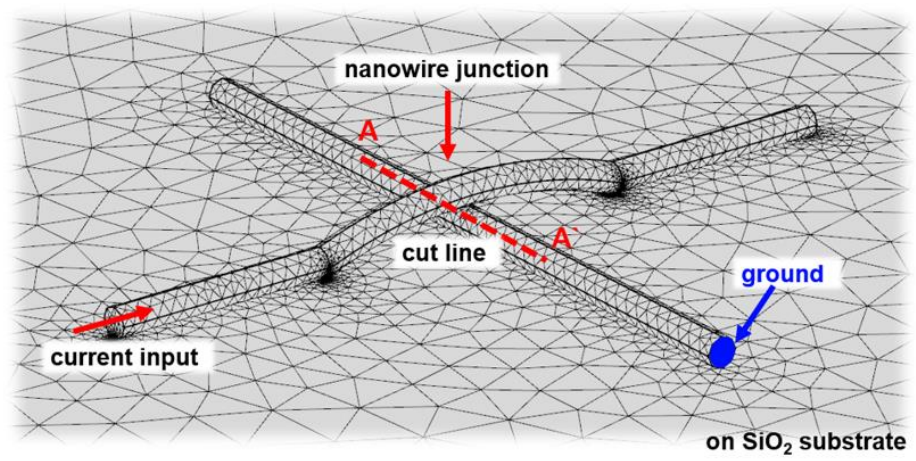

(b)

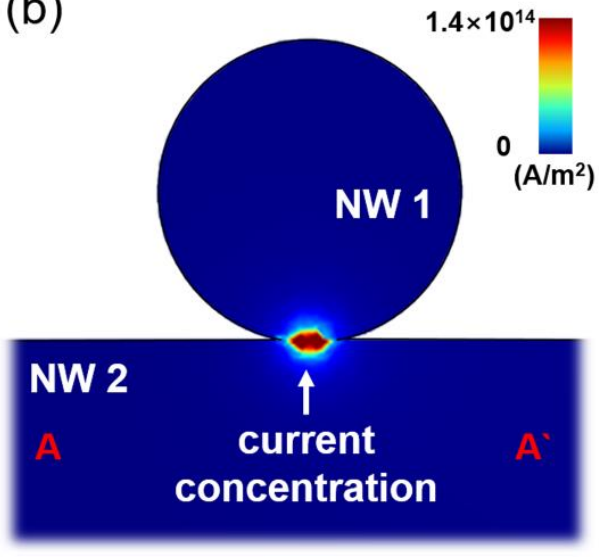

(c) $\mathrm{T}_{\text {surface }} / \mathrm{T}_{\max }: \sim 93 \%$

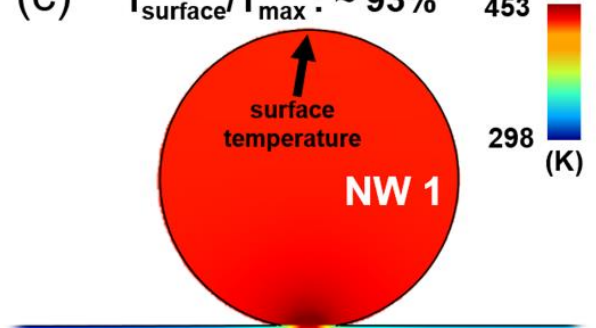

\section{NW 2}

453 


\section{SI References}

(S1) Kim, K.; Chung, J.; Hwang, G.; Kwon, O.; Lee, J. Quantitative Measurement with Scanning Thermal Microscope by Preventing the Distortion Due to the Heat Transfer through the Air. ACS Nano 2011, 5, 8700-8709.

(S2) Choi, D.; Poudel, N.; Park, S.; Akinwande, D.; Cronin, S. B.; Watanabe, K.; Taniguchi, T.; Yao, Z.; Shi, L. Large Reduction of Hot Spot Temperature in Graphene Electronic Devices with Heat-Spreading Hexagonal Boron Nitride. ACS Appl. Mater. Interfaces 2018, 10, 11101-11107.

(S3) Das, S. R.; Mohammed, A. M. S.; Maize, K.; Sadeque, S.; Shakouri, A.; Janes, D. N.; Alam, M. A. Evidence of Universal Temperature Scaling in Self-Heated Percolating Networks. Nano Lett 2016, 16, 3130-3136.

(S4) Jeong, W.; Kim, K.; Kim, Y.; Lee, W.; Reddy, P. Characterization of nanoscale temperature fields during electromigration of nanowires. Scientific Reports 2014, 4, 4975-4979. 